\title{
STABILITY OF A PROCESSOR-SHARING QUEUE WITH VARYING THROUGHPUT
}

\author{
PASCAL MOYAL, ${ }^{*}$ UTC Compiègne
}

\begin{abstract}
In this paper we present a stability criterion for processor-sharing queues, in which the throughput may depend on the number of customers in the system (such as in the case of interferences between users). Such a system is represented by a point measure-valued stochastic recursion keeping track of the remaining processing times of the customers.
\end{abstract}

Keywords: Queueing system; stability; measure-valued process; stochastic recurrence; processor sharing

2000 Mathematics Subject Classification: Primary 60F17

Secondary 60K25; 60B 12

\section{Introduction}

In this paper we address the question of stationarity in the general ergodic framework for processor-sharing queues, in which the throughput (i.e. the quantity of work achieved by the server(s) per unit of time) may depend on the state of the system. More precisely, we assume hereafter that the server(s) (it will be clear in the sequel that the effective number of servers does not really matter, only the quantity of work consumed per unit of time matters) processes all the jobs present in the system simultaneously and fairly. Whenever there are $n$ customers in the system, each of them is thus served at a rate that depends on $n$, say $r(n)$. The classical case is when $r(n)=1 / n, n \geq 1$, so that the total throughput equals $n r(n)=1$ whenever the system is nonempty: this is the classical processor-sharing queue. Hereafter, we consider a more general context in which the total throughput may decrease with the number of customers in the system (hence, $n r(n) \leq 1$ ). This is the case, for instance, in a wireless network in which the number of users currently active may decrease the efficiency of the resources. Another case is when the value of $n$, the number of customers, does not change the nominal service rate $r(n)$, say $r(n)=1$ for all $n$. This corresponds to the classical queue with infinitely many servers.

In both cases and under general stationary ergodic assumptions, Loynes' stability result does not hold, since this is not a proper G/G/1 queue (the throughput may be less or larger than 1). We address the question of the existence of a stationary version of such queues by representing them with point measure-valued stochastic recursions in the Palm setting, so as to take into account the dependency on the number of customers. These point measures keep track of all the remaining service times of all the customers in the system. Then it is possible to provide conditions for the existence of a stationary version of this sequence, which allows us to explicitly construct stationary queues under these assumptions.

This paper is organized as follows. After some preliminaries in Section 2, we present the queueing models we consider in Section 3. In Section 4 we study the particular case of the

Received 4 April 2008; revision received 2 October 2008.

* Postal address: Laboratoire de Mathématiques Appliquées de Compiègne, Université de Technologie de Compiègne, Département Génie Informatique, Centre de Recherches de Royallieu, BP 20 529, 60205 Compiegne Cedex, France. Email address: moyalpas@dma.utc.fr 
G/G/ $\infty$ queue, and in Section 5 we present a stability criterion for generalized processor queues with state-dependent throughput.

\section{Preliminaries}

Let $M_{f}^{+}$and $\mathcal{C}_{\mathrm{b}}$ respectively denote the set of positive finite measures on $\mathbb{R}_{+}^{*}$ and the set of bounded continuous functions from $\mathbb{R}$ to $\mathbb{R}$. Equipped with the weak topology $\sigma\left(M_{f}^{+}, \mathcal{C}_{\mathrm{b}}\right)$, $M_{f}^{+}$is a Polish space (see [2]). Let $\tilde{0}$ be the zero measure on $\mathbb{R}$ (i.e. such that $\tilde{0}(\mathfrak{B})=0$ for any Borel set $\mathfrak{B}$ on $\mathbb{R}$ ). For any $\mu \in M_{f}^{+}$and any measurable $f: \mathbb{R} \rightarrow \mathbb{R}$, we classically write $\langle\mu, f\rangle:=\int f \mathrm{~d} \mu$. For any $y \in \mathbb{R}$ and any measurable $f: \mathbb{R} \rightarrow \mathbb{R}$, let $\tau_{y} f(\cdot)=f(\cdot-y) \mathbf{1}_{\{\cdot>y\}}$. Then, for any $\mu \in M_{f}^{+}, \tau_{y} \mu$ denotes the only element of $M_{f}^{+}$such that $\left\langle\tau_{y} \mu, f\right\rangle=\left\langle\mu, \tau_{y} f\right\rangle$.

Let the set $M_{f}^{+}$be endowed with the increasing partial integral order, ' $\preceq$ ': for any two $\mu, v \in M_{f}^{+}, \mu \preceq v$ if $\langle\mu, f\rangle \leq\langle\tilde{\sim}, f\rangle$ for any measurable nondecreasing function $f$ such that these integrals exist. Of course, $\tilde{0} \preceq \mu$ for any $\mu \in M_{f}^{+}$. Furthermore, we note the following lemma.

Lemma 1. Any sequence of $M_{f}^{+}$that is ' weak topology.

Proof. Let $\left\{\mu_{n}\right\}_{n \in \mathbb{N}}$ be a ' $\preceq$ '-increasing sequence of $M_{f}^{+}$that is bounded above by $\mu \in M_{f}^{+}$. Then, as easily seen, the sequence of nonincreasing real functions, $\left\{\mu_{n}([\cdot, \infty))\right\}_{n \in \mathbb{N}}$, tends pointwise and, hence (this is the Diniz theorem), uniformly to a nonincreasing real function $f$ that is right continuous and has a countable number of discontinuities. Moreover, $f(0) \leq$ $\mu\left(\mathbb{R}_{+}^{*}\right)<\infty$, and we can fully characterize a measure $\mu^{*} \in M_{f}^{+}$setting $\mu^{*}((0, x))=f(0)-$ $f(x)$ for all $x \in \mathbb{R}_{+}^{*}$. In particular, $\sup _{x \in \mathbb{R}_{+}^{*}}\left|\mu^{n}((0, x))-\mu^{*}((0, x))\right| \longrightarrow 0$ as $n \rightarrow \infty$; hence, $\mu^{n}$ tends to $\mu^{*}$ in total variation. This completes the proof.

Now let $\mathcal{M} \subset M_{f}^{+}$be the subset of finite (simple) counting measures on $\mathbb{R}_{+}^{*}$. Any $\mu \in$ $\mathcal{M} \backslash\{\tilde{0}\}$ reads $\mu=\sum_{i=1}^{N(\mu)} \delta_{\alpha_{i}(\mu)}$, where $N(\mu):=\mu\left(\mathbb{R}_{+}^{*}\right)$ is the number of atoms of $\mu$, $\delta_{x}$ is the Dirac measure at $x \in \mathbb{R}+$, and $\alpha_{1}(\mu)<\alpha_{2}(\mu)<\cdots<\alpha_{N(\mu)}(\mu)$. Then, $\tau_{y}(\mu)=\sum_{i=1}^{N(\mu)} \delta_{\alpha_{i}(\mu)-y} \mathbf{1}_{\left\{\alpha_{i}(\mu)>y\right\}}$ and, for any two $\mu, v \in \mathcal{M} \backslash\{\tilde{0}\}, \mu \preceq v$ whenever

(i) $N(\mu) \leq N(v)$,

(ii) for all $i=0, \ldots, N(\mu)-1, \alpha_{N(\mu)-i}(\mu) \leq \alpha_{N(v)-i}(\nu)$.

For any $\mu \in \mathcal{M} \backslash\{\tilde{0}\}$, let $Z(\mu)=\alpha_{N(\mu)}(\mu)$, the largest atom of $\mu$. Finally, we write $x^{+}=$ $\max \{x, 0\}$ for any real number $x$, and $\sum_{i=j}^{k} \cdot \equiv 0$ whenever $k<j$ and $\max \{\varnothing\} \equiv 0$.

\section{The model}

Let us first introduce our definitions and assumptions on the queueing systems we will consider in the sequel. Let $\left(\Omega, \mathcal{F}, \mathrm{P}, \theta_{t}\right)$ be a probability space furnished with a bijective flow $\left(\theta_{t}\right)_{t \geq 0}$, under which $\mathrm{P}$ is stationary and ergodic. Define on $\Omega$ the $\theta_{t}$-compatible simple point process $\left(A_{t}\right)_{t \in \mathbb{R}}$ of points $\cdots<T_{-2}<T_{-1}<T_{0} \leq 0<T_{1}<T_{2}<\cdots$, which represent the arrival times of the customers in a queue without a buffer. The process $\left(A_{t}\right)_{t \in \mathbb{R}}$ is marked by a sequence $\left\{\sigma_{n}\right\}_{n \in \mathbb{Z}}$, where, for all $n \in \mathbb{Z}, \sigma_{n}$ is the service duration requested by customer $C_{n}$ who arrived at time $T_{n}$. Also, for all $n \in \mathbb{Z}$, let $\xi_{n}=T_{n+1}-T_{n}$, and suppose that the generic random variables (RVs) $\sigma$ and $\xi$ are integrable. We consider servers that follow a generalized processor-sharing discipline. By this we mean that all present customers are taken care of simultaneously at a rate $r$, which is equal for all customers. An example is of 
TABle 1.

\begin{tabular}{ccc}
\hline Number of customers & Nominal service rate & Throughput \\
\hline 1 & 1.000 & 1.00 \\
2 & 0.495 & 0.99 \\
3 & 0.300 & 0.90 \\
$\vdots$ & $\vdots$ & $\vdots$ \\
100 & 0.008 & 0.80 \\
\hline
\end{tabular}

course provided by the classical processor-sharing queue, but it will be shown in the subsequent sections that significant results can also be obtained for a wider class of systems. Indeed, in many cases, it is plausible to assume that the amount of work in the system might affect the throughput, considering, for instance, the working cost induced by the switching mechanism in the processor or the interferences between the users of a wireless network. In both cases, it is then natural to assume that the rate $r$ is a nonincreasing function of the service profile, i.e. $\mu \preceq v$ implies that $r(\mu) \geq r(v)$. Hereafter, for the sake of simplicity, we will restrict our attention to the subcase, where $r$ is a nonincreasing function of the number of customers in the system, although it should be clear that all the results below also hold when $r$ is a function of the whole service profile. In other words, at any $t$, each customer is allocated a quantity of work, $r\left(Q_{t}\right)$, per unit of time, where $Q(t)$ denotes the number of customers in the system at $t$, that is, $r(i) \geq r(j)$ for all $i, j \in \mathbb{N}^{*}$ such that $i \leq j$. In Table 1 we illustrate, through a naive example, the effect of a large number of customers on the throughput.

Provided that $C_{n}$ is in the system at $t$, his remaining processing time at this instant is the time before his service completion. The service profile of the system at $t$ is the $\mathcal{M}$-valued process keeping track of the remaining processing times of all the customers in the system at $t$ :

$$
\mu(t)=\sum_{i=1}^{Q(t)} \delta_{\alpha_{i}(\mu(t))},
$$

where $\alpha_{1}(\mu(t)) \leq \alpha_{2}(\mu(t)) \leq \cdots \leq \alpha_{Q(t)}(\mu(t))$ denote the remaining processing times of the customers in the system at $t$, ranked in decreasing order. Let $W(t)$ denote the workload at $t$. Then the workload and the congestion processes can be recovered easily from the service profile process by writing, for all $t$,

$$
Q(t)=N(\mu(t)), \quad W(t)=\langle\mu(t), I\rangle,
$$

where $I$ is the identity function. The processes $\mu, Q$, and $W$ have càdlàg paths (i.e. paths that are continuous from the right with left limits), and, for all $t$, let $\mu(t-)=\lim _{s \uparrow \uparrow t} \mu(s)$ (and similarly for $Q(t-)$ and $W(t-))$. For all $n \in \mathbb{N}$, we respectively denote by $\mu_{n}=\mu\left(T_{n}-\right)$, $Q_{n}=Q\left(T_{n}-\right)$, and $W_{n}=X\left(T_{n}-\right)$ the service profile, the congestion, and the workload just before the arrival of customer $C_{n}$.

Let $\left(\Omega, \mathcal{F}, \mathrm{P}^{0}\right)$ be the Palm space of $A$, let $\theta:=\theta_{T_{1}}$, let $\theta^{-1}$ be its measurable inverse, and, for all $n \in \mathbb{Z}$, let $\theta^{n}=\theta \circ \theta \circ \cdots \circ \theta$ and $\theta^{-n}=\theta^{-1} \circ \theta^{-1} \circ \cdots \circ \theta^{-1}$. Note that $\mathrm{P}^{0}$ is stationary and ergodic under $\theta$, i.e. for all $\mathfrak{A} \in \mathcal{F}, \mathrm{P}^{0}\left[\theta^{-1} \mathfrak{A}\right]=\mathrm{P}^{0}[\mathfrak{A}]$ and $\theta \mathfrak{A}=\mathfrak{A}$ implies that $\mathrm{P}^{0}[\mathfrak{A}]=0$ or 1 and that all $\theta$-contracting events (such that $\mathrm{P}^{0}\left[\mathfrak{A}^{c} \cap \theta^{-1} \mathfrak{A}\right]=0$ ) are $\theta$-invariant. Letting $\xi:=\xi_{0}$ and $\sigma:=\sigma_{0}$, we have, for all $n \in \mathbb{Z}, \xi_{n}:=\xi \circ \theta^{n}$ and $\sigma_{n}:=\sigma \circ \theta^{n}$. 
We say that the $E$-valued random sequence $\left\{X_{n}\right\}_{n \in \mathbb{N}}$ is a stochastically recursive sequence (SRS) whenever, for some random mapping $\phi: E \rightarrow E$,

$$
X_{n+1}=\phi \circ \theta^{n}\left(X_{n}\right), \quad n \in \mathbb{N}, \mathrm{P}^{0} \text {-almost surely }\left(\mathrm{P}^{0} \text {-a.s. }\right) .
$$

For any $E$-valued RV $Y$, let $\left\{X_{n}^{[Y]}\right\}_{n \in \mathbb{N}}$ be the SRS $\left\{X_{n}\right\}_{n \in \mathbb{N}}$ such that $X_{0}^{[Y]}=Y$, $\mathrm{P}^{0}$-a.s. We follow the formalism of [1] and formulate the question of stationarity for the $\operatorname{SRS}\left\{X_{n}\right\}_{n \in \mathbb{N}}$ in the following terms. There exists a stationary version of $\left\{X_{n}\right\}_{n \in \mathbb{N}}$ whenever, for some $Y$ and all $n, X_{n}^{[Y]}=Y \circ \theta^{n}, \mathrm{P}^{0}$-a.s., or, in other words, provided that the equation

$$
Y \circ \theta=\phi(Y)
$$

admits a solution that is an $E$-valued RV. We say that two sequences of RVs $\left\{X_{n}\right\}_{n \in \mathbb{N}}$ and $\left\{Y_{n}\right\}_{n \in \mathbb{N}}$ couple provided that

$$
\mathrm{P}^{0}\left[\text { there exists } N(\omega), X_{n}(\omega)=Y_{n}(\omega) \text { for all } n \geq N(\omega)\right]=1
$$

and that there is strong backwards coupling from $\left\{X_{n}\right\}_{n \in \mathbb{N}}$ with the stationary sequence $\left\{Y \circ \theta^{n}\right\}$ whenever

$$
\mathrm{P}^{0}\left[\text { there exists } N^{\prime}(\omega), X_{n} \circ \theta^{-n}(\omega)=Y(\omega) \text { for all } n \geq N^{\prime}(\omega)\right]=1 .
$$

Lemma 2. The sequence $\left\{\mu_{n}\right\}_{n \in \mathbb{N}}$ is stochastically recursive for any rate function $r$ : letting, for all $\mu \in \mathcal{M}$ and $x \in \mathbb{R}_{+}^{*}$,

- for all $i \leq N(\mu)$,

$$
\gamma_{i}^{r}(\mu, x)=r(N(\mu)-i+1)\left(x-\sum_{j=1}^{i-1} \alpha_{j}(\mu)\left(\frac{1}{r(N(\mu)-j+1)}-\frac{1}{r(N(\mu)-j)}\right)\right),
$$

- $i^{r}(\mu, x)=\max \left\{i \leq N(\mu) ; \alpha_{i}(\mu) \leq \gamma_{i}^{r}(\mu, x)\right\}$,

- $\gamma^{r}(\mu, x):=\gamma_{\left(i^{r}(\mu, x)+1\right) \wedge 1}^{r}(\mu, x)$,

- $\Phi^{r}(\mu, x)=\tau_{\gamma^{r}(\mu, x)} \mu$,

we have, for any initial profile $\mu_{0}$ and all $n \in \mathbb{N}$,

$$
\mu_{n+1}=\Phi^{r}\left(\mu_{n}+\delta_{\sigma_{n}}, \xi_{n}\right) .
$$

Proof. Just after the arrival of $C_{n}$, the service profile reads $\mu:=\mu_{n}+\delta_{\sigma_{n}}$. Set $T_{0}^{\prime}:=T_{n}$ and $\alpha_{0}(\mu)=0$. For any $i \in\{1, \ldots, N(\mu)\}$, let $T_{i}^{\prime}$ be the theoretical departure of customer $\tilde{C}_{i}$ whose remaining service time at $T_{n}$ is $\alpha_{i}(\mu)$. The remaining service time of $\tilde{C}_{i}$ at $T_{i-1}^{\prime}$ is $\alpha_{i}(\mu)-\alpha_{i-1}(\mu)$, and between $T_{i-1}^{\prime}$ and $T_{i}^{\prime}, \tilde{C}_{i}$ is served at rate $r(N(\mu)-i+1)$. Hence, we have the induction formula

$$
T_{i}^{\prime}=T_{i-1}^{\prime}+\frac{\alpha_{i}(\mu)-\alpha_{i-1}(\mu)}{r(N(\mu)-i+1)}, \quad i \in\{1, \ldots, N(\mu)\},
$$

from which we deduce, for all $i \in\{1, \ldots, N(\mu)\}$,

$$
T_{i}^{\prime}=T_{n}+\frac{\alpha_{i}(\mu)}{r(N(\mu)-i+1)}+\sum_{j=1}^{i-1} \alpha_{j}(\mu)\left(\frac{1}{r(N(\mu)-j+1)}-\frac{1}{r(N(\mu)-j)}\right) .
$$


For any $i$, customer $\tilde{C}_{i}$ leaves the system before $T_{n+1}$ provided that $T_{i}^{\prime}-T_{n} \leq \xi_{n}$, which is equivalent to $\alpha_{i}(\mu) \leq \gamma_{i}^{r}\left(\mu, \xi_{n}\right)$ in view of (3). In particular, $i^{r}\left(\mu, \xi_{n}\right)$ denotes the index of the last customer leaving the system before $T_{n+1}$ (or 0 if there is no departure between $T_{n}$ and $\left.T_{n+1}\right)$. Then the system is not empty at $T_{n+1}-$ provided that $i^{r}\left(\mu, \xi_{n}\right)<N(\mu)$, and in this case, $\left\{\tilde{C}_{i}, i \in\left\{i^{r}\left(\mu, \xi_{n}\right)+1, N(\mu)\right\}\right\}$ is the set of customers present in the system at $T_{n+1}-$. For such $i>i^{r}\left(\mu, \xi_{n}\right)$, the remaining service time of $\tilde{C}_{i}$ at $T_{n+1}$ is given by

$$
\alpha_{i}(\mu)-\alpha_{i^{r}\left(\mu, \xi_{n}\right)}(\mu)-r\left(N(\mu)-i^{r}\left(\mu, \xi_{n}\right)\right)\left(T_{n+1}-T_{i^{r}\left(\mu, \xi_{n}\right)}^{\prime}\right)=\alpha_{i}(\mu)-\gamma^{r}\left(\mu, \xi_{n}\right) .
$$

Thus, functional mapping of the profile at $T_{n}$ onto the profile at $T_{n+1}-$ reads

$$
\Phi^{r}\left(\cdot, \xi_{n}\right): \mu \longmapsto \sum_{i=i^{r}\left(\mu, \xi_{n}\right)+1}^{N(\mu)} \delta_{\alpha_{i}(\mu)-\gamma^{r}\left(\mu, \xi_{n}\right)} .
$$

To obtain the announced result, we note that, for any $\mu \in \mathcal{M}, x \in \mathbb{R}_{+}^{*}$, and any $i<N(\mu)$, we have

$$
\gamma_{i+1}^{r}(\mu, x)-\gamma_{i}^{r}(\mu, x)=\frac{r(N(\mu)-i)-r(N(\mu)-i+1)}{r(N(\mu)-i+1)}\left(\gamma_{i}^{r}(\mu, x)-\alpha_{i}(\mu)\right),
$$

which is nonnegative if and only if $i \leq i^{r}(\mu, x)$. Hence,

$$
\gamma^{r}(\mu, x)=\max _{1 \leq i \leq N(\mu)} \gamma_{i}^{r}(\mu, x)
$$

and, in particular, $\Phi^{r}\left(\mu, \xi_{n}\right)=\tau_{\gamma^{r}}\left(\mu, \xi_{n}\right) \mu, \mathrm{P}^{0}$-a.s.

For a fixed $x \in \mathbb{R}+$, the two following monotonicity properties of the mappings $\Phi^{r}(\cdot, x)$ hold, as shown in Appendix A.

Lemma 3. For any $x \in \mathbb{R}+$ and any rate function $r$, the mapping $\Phi^{r}(\cdot, x)$ is nondecreasing from $\mathcal{M}$ into itself.

Lemma 4. For any $x \in \mathbb{R}+$ and any $\mu \in \mathcal{M}$, and for any two rate functions $r$ and $\tilde{r}$ such that $r(i) \leq \tilde{r}(i)$ for all $i \in \mathbb{N}^{*}, \Phi^{r}(\mu, x) \succeq \Phi^{\tilde{r}}(\mu, x)$.

\section{The pure delay system}

Let us first consider the case where the rate function is constant with respect to the size of the system, say $r(i)=1$ for any $i \geq 1$. This corresponds to the classical 'pure delay' G/G/ $\infty$ queue: all present customers are simultaneously served at unit rate and, hence, spend a time equal to their service duration in the system, which is equivalent to saying that there is an infinite number of servers. In this case, the recursive equation (1) driving the service profile sequence (for which a diffusion approximation is given in [5] in the $\mathrm{M} / \mathrm{GI} / \infty$ case) specializes to

$$
\mu_{n+1}=\tau_{\xi_{n}}\left(\mu_{n}+\delta_{\sigma_{n}}\right)
$$

and a stationary service profile for the queue is a solution to the equation

$$
\mu \circ \theta=\tau_{\xi}\left(\mu+\delta_{\sigma}\right)
$$

The following lemma (see [7]) will be used in the sequel. 
Lemma 5. There exists a unique $\mathrm{P}^{0}$-a.s. finite solution to the equation

$$
L \circ \theta=[\max \{L, \sigma\}-\xi]^{+}
$$

given by

$$
L:=\left[\sup _{j \in \mathbb{N}^{*}}\left(\sigma_{-j}-\sum_{i=1}^{j} \xi_{-i}\right)\right]^{+}
$$

Proof. Existence. Loynes' theorem for stochastic recurrences (see [1, p. 107] and [6]) can be applied since the mapping $x \mapsto[\max \{x, \sigma\}-\xi]^{+}$is $\mathrm{P}^{0}$-a.s. continuous and nondecreasing. The minimal solution $L$ to (6) classically reads as the $\mathrm{P}^{0}$-almost sure limit of Loynes's sequence $\left\{L_{n}^{[0]} \circ \theta^{-n}\right\}_{n \in \mathbb{N}}$, where $\left\{L_{n}^{[0]}\right\}_{n \in \mathbb{N}}$ is the initially null SRS that is defined by

$$
L_{n+1}^{[0]}=\left[\max \left\{L_{n}^{[0]}, \sigma_{n}\right\}-\xi_{n}\right]^{+} \quad \text { for all } n \in \mathbb{N} .
$$

It is routine to check from Birkhoff's ergodic theorem (and the fact that $\sigma$ is not identically 0 ) that $L$ is $\mathrm{P}^{0}$-a.s. finite.

Uniqueness. Let $\tilde{L}$ be a solution to (6). First, note that if $\tilde{L}>\sigma, \mathrm{P}^{0}$-a.s. would imply that, on a $\mathrm{P}^{0}$-a.s. event,

$$
\tilde{L} \circ \theta>0 \Longleftrightarrow \tilde{L} \circ \theta=\tilde{L}-\xi
$$

a contradiction to the ergodic lemma. Hence, in view of the minimality of $L$ we have

$$
\mathrm{P}^{0}[\tilde{L}=L]=\mathrm{P}^{0}[\tilde{L} \circ \theta \leq L \circ \theta] \geq \mathrm{P}^{0}[\tilde{L} \leq \sigma]>0,
$$

which implies that $\{\tilde{L}=L\}$ is $\mathrm{P}^{0}$-almost sure since it is $\theta$-contracting.

We can now state the following result.

Theorem 1. Equation (5) admits a finite solution, given by

$$
\mu^{\infty}=\sum_{i=1}^{\infty} \delta_{\left(\sigma_{-i}-\sum_{j=1}^{i} \xi_{-j}\right)} \mathbf{1}_{\left\{\sigma_{-i} \geq \sum_{j=1}^{i} \xi_{-j}\right\}} .
$$

Moreover, provided that

$$
\mathrm{P}^{0}[L \leq 0]>0
$$

this solution is unique and, for all $\zeta$ such that $Z(\zeta) \leq L, \mathrm{P}^{0}$-a.s., the sequence $\left\{\mu_{n}^{[\zeta]}\right\}_{n \in \mathbb{N}}$ converges with strong backwards coupling to $\mu^{\infty}$.

Proof. Existence. It is a straightforward consequence of Birkhoff's ergodic theorem that

$$
\mathrm{P}^{0}\left[\mu^{\infty} \in \mathcal{M}\right]=\mathrm{P}^{0}\left[\operatorname{card}\left\{i \in \mathbb{N}^{*}, \sigma_{-i}-\sum_{j=1}^{i} \xi_{-j} \geq 0\right\}<\infty\right]>0
$$

This $\theta$-contracting event is thus $\mathrm{P}^{0}$-almost sure. On the other hand, in view of Lemma 3, the mapping $\mu \mapsto \tau_{\xi}\left(\mu+\delta_{\sigma}\right)$ is $\mathrm{P}^{0}$-a.s. nondecreasing from $\mathcal{M}$ into itself. Furthermore, it 
is continuous for the weak topology, as easily checked from the fact that, for any $\mathcal{M}$-valued sequence $\left\{v_{n}\right\}_{n \in \mathbb{N}}$ tending weakly to $v$, any $x, s \in \mathbb{R}+$, and any $\phi \in \mathcal{C}_{\mathrm{b}}$,

$$
\begin{aligned}
\left\langle\tau_{x} v_{n}+\delta_{s}, \phi\right\rangle & =\int \phi(y-x) \mathrm{d} v_{n}(y)+\phi(s) \\
& \rightarrow \int \phi(y-x) \mathrm{d} v(y) \quad \text { as } n \rightarrow \infty \\
& =\left\langle\tau_{x} v+\delta_{s}, \phi\right\rangle .
\end{aligned}
$$

Thus, we can follow the steps of Loynes' construction (Lemma 1) to conclude that $\mu^{\infty}$ is the ' $\preceq$ '-minimal solution of (5) since it is the $\mathrm{P}^{0}$-almost sure limit of the sequence given, for all $n \in \mathbb{N}$, by

$$
\mu_{n}^{[\tilde{0}]} \circ \theta^{-n}=\sum_{i=1}^{\infty} \delta_{\left(\sigma_{-i}-\sum_{j=1}^{i} \xi_{-j}\right)} \mathbf{1}_{\left\{\sigma_{-i} \geq \sum_{j=1}^{i} \xi_{-j}\right\}} .
$$

Uniqueness. It is easily checked that, for any solution $\mu$ of (5),

$$
Z(\mu) \circ \theta=Z\left(\tau_{\xi}\left(\mu+\delta_{\sigma}\right)\right)=[\max \{Z(\mu), \sigma\}-\xi]^{+}
$$

hence, $Z(\mu)=L, \mathrm{P}^{0}$-a.s. Moreover, since $\mu^{\infty}$ is the minimal solution of (5), we have

$$
\left\{\mu=\mu^{\infty}\right\} \supseteq\{\mu=\tilde{0}\}=\{Z(\mu)=0\}=\{L=0\} .
$$

Hence, whenever (7) holds, the event $\left\{\mu=\mu^{\infty}\right\}$ has a positive probability. Since it is $\theta$-invariant, it is $\mathrm{P}^{0}$-almost sure.

Coupling. Let $\zeta$ be an $\mathcal{M}$-valued RV such that $Z(\zeta) \leq L, \mathrm{P}^{0}$-a.s. It is easy to construct another $\mathcal{M}$-valued $\operatorname{RV} \tilde{\zeta}$ such that $\zeta \preceq \tilde{\zeta}$ and $Z(\tilde{\zeta})=\bar{L}$, $\mathrm{P}^{0}$-a.s. by setting, for example, $\tilde{\zeta}=\sum_{i=1}^{N(\zeta)-1} \delta_{i}(\zeta)+\delta_{L}$. From Lemma 3, it follows by induction that $\mu_{n}^{[\zeta]} \preceq \mu_{n}^{[\tilde{\zeta}]}, \mathrm{P}^{0}$-a.s. for all $n \in \mathbb{N}$. Now note that, for all $n \in \mathbb{N}, Z\left(\mu_{n}^{[\tilde{\zeta}]}\right)=L \circ \theta^{n}$, as is easily checked by induction. Hence, for all $n \in \mathbb{N}$, we have

$$
\mathcal{E}_{n}:=\left\{L \circ \theta^{n}=0\right\}=\left\{Z\left(\mu_{n}^{[\tilde{\zeta}]}\right)=0\right\}=\left\{\mu_{n}^{[\tilde{\zeta}]}=\tilde{0}\right\} \subseteq\left\{\mu_{n}^{[\zeta]}=\tilde{0}\right\} .
$$

Therefore, $\left\{\varepsilon_{n}\right\}_{n \in \mathbb{N}}$ is a stationary sequence of renovating events of length 1 for $\left\{\mu_{n}^{[\zeta]}\right\}_{n \in \mathbb{N}}$ (see [3] and [4]) for any $\zeta$ such that $Z(\zeta) \leq L, \mathrm{P}^{0}$-a.s. Assumption (7) implies the coupling property for such an initial condition in view of Corollary 2.5.1 of [1].

As simple consequences of the latter result, let us note the following coupling properties.

Corollary 1. Under condition (7), for any $\zeta$ such that $Z(\zeta) \leq L, \mathrm{P}^{0}$-a.s.,

(i) $\left\{X_{n}^{[N(\zeta)]}\right\}_{n \in \mathbb{N}}$ converges with strong backwards coupling to $N\left(\mu^{\infty}\right)$,

(ii) $\left\{W_{n}^{[\langle\zeta, I\rangle]}\right\}_{n \in \mathbb{N}}$ converges with strong backwards coupling to $\left\langle\mu^{\infty}, I\right\rangle$.

\section{Processor-sharing queues}

We will now consider the case where the rate function depends on the number of customers in the system at the current time. We assume hereafter that the nondecreasing function $r$ is such 
that

$$
\begin{gathered}
\sup _{n \in \mathbb{N}^{*}} n r(n) \leq 1, \\
K_{r}=\inf _{n \in \mathbb{N}^{*}} n r(n)>0 .
\end{gathered}
$$

Assumption (8) amounts to saying that there is a single server, since the throughput at time $t$, given by $Q(t) r(Q(t))$, may not exceed 1. A typical case is the classical processor-sharing queue: assume that $r(n)=n^{-1}$ for any $n$ (and, hence, $K_{r}=1$ ), meaning that all customers are served at a rate that is inversely proportional to the number of customers. In this case the server works at unit rate whatever the number of customers in the system. Whenever $K_{r}<1$, the number of customers affects the velocity of service, so that the total throughput may be less than 1. Nevertheless, we assume in (9) that a minimal throughput $K_{r}$ is granted for a given $r$, i.e. the server always achieves at least $K_{r}$ units of work per unit of time. An example is provided by the following idealistic scenario: the server works at unit rate whenever there is only one customer in the system $(r(1)=1)$, and, when there are several customers in service at the same time, the interferences (or operating cost) decrease the efficiency of the server by half, so that $r(i)=1 / 2 i$ for any $i \geq 2$, which implies in particular that (9) is satisfied for $K_{r}=\frac{1}{2}$.

In view of Lemma 2, a stationary service profile is a solution to the equation

$$
\mu \circ \theta=\Phi^{r}\left(\mu+\delta_{\sigma}, \xi\right) .
$$

We have the following result.

Theorem 2. Let $r$ be a rate function satisfying assumptions (8) and (9). Then, provided that

$$
\mathrm{E}^{0}[\sigma]<K_{r} \mathrm{E}^{0}[\xi]
$$

(10) admits a unique finite solution $\mu^{r}$. Moreover, for any $\mathcal{M}$-valued $R V \zeta$ such that $\langle\zeta, I\rangle \leq$ $W^{K_{r}}, \mathrm{P}^{0}$-a.s. (where $W^{K_{r}}$ is the unique solution of $(12)$, below), the sequence $\left\{\mu_{n}^{[\zeta]}\right\}_{n \in \mathbb{N}}$ converges with strong backwards coupling to $\mu^{r}$.

Proof. Existence. Fix $r$ to satisfy assumptions (8) and (9). From Loynes's fundamental stability result, the equation

$$
W \circ \theta=\left[W+\sigma-K_{r} \xi\right]^{+}
$$

admits a unique $\mathrm{P}^{0}$-a.s. finite solution, say $W^{K_{r}}$, provided that (11) holds. Let $\tilde{r}$ be the rate function such that, for all $\mu \in \mathcal{M}, \tilde{r}(\mu)=K_{r} / N(\mu)$, so that the throughput under $\tilde{r}$ always equals $K_{r}$ whenever the system is nonempty. Let $\zeta$ be an $\mathcal{M}$-valued RV such that $\langle\zeta, I\rangle \leq W^{K_{r}}$ and

$$
\tilde{\zeta}=\zeta+\delta_{W^{K_{r}-\langle\zeta, I\rangle}} \mathbf{1}_{\left\{W^{K_{r}}>\langle\zeta, I\rangle\right\}}
$$

It is then clear that $\langle\tilde{\zeta}, I\rangle=W^{K_{r}}$. Moreover, we have, $\mathrm{P}^{0}$-a.s. for all $n \in \mathbb{N}$,

$$
\left\langle\mu_{n+1}^{\tilde{r},[\tilde{\zeta}]}, I\right\rangle=\left[\left\langle\mu_{n}^{\tilde{r},[\tilde{\zeta}]}, I\right\rangle+\sigma_{n}-K_{r} \xi_{n}\right]^{+},
$$

as the throughput equals $K_{r}$ at any time (as is easily checked from Lemma 2), so that $\left\langle\mu_{n+1}^{\tilde{r},[\tilde{\zeta}]}, I\right\rangle=W^{K_{r}} \circ \theta^{n}$ for all $n \in \mathbb{N}$. On the other hand, $\zeta \preceq \tilde{\zeta}$; hence, in view of Lemmas 3 and 4 , an immediate induction shows that $\mu_{n}^{r,[\zeta]} \preceq \mu_{n}^{\tilde{r} \text {, }[\tilde{\zeta}]}$ for all $n \in \mathbb{N}$, which in turn implies that

$$
\left\langle\mu_{n}^{r,[\zeta]}, I\right\rangle \leq\left\langle\mu_{n}^{\tilde{r},[\tilde{\zeta}]}, I\right\rangle=W^{K_{r}} \circ \theta^{n} \quad \text { for all } n \in \mathbb{N} .
$$


Therefore, for all $n \in \mathbb{N}$, on $\mathfrak{A}_{n}:=\left\{W^{K_{r}} \circ \theta^{n}=0\right\}$, we have $\left\langle\mu_{n}^{r,[\zeta]}, I\right\rangle=0$; hence,

$$
\mu_{n}^{r,[\zeta]}=\tilde{0} \quad \text { and } \quad \mu_{n+1}^{r,[\zeta]}=\Phi^{r}\left(\delta_{\sigma_{n}}, \xi_{n}\right) .
$$

Therefore, $\left\{\mu_{n}^{r,[\zeta]}\right\}_{n \in \mathbb{N}}$ admits $\left\{\mathfrak{A}_{n}\right\}_{n \in \mathbb{N}}$ as a stationary sequence of renovating events of length 1 . Furthermore, the event $\mathfrak{A}_{0}=\left\{W^{K_{r}}=0\right\}$ has a strictly positive probability, since the contrary would imply that

$$
\mathrm{E}^{0}\left[W^{K_{r}} \circ \theta-W^{K_{r}}\right]=\mathrm{E}^{0}\left[\sigma-K_{r} \xi\right]<0,
$$

an absurdity in view of the ergodic lemma. Then it follows from [1, Theorem 2.5.3] that there is strong backwards coupling of $\mu_{n}^{r,[\zeta]}$ with the stationary sequence $\left\{\mu^{r} \circ \theta^{n}\right\}_{n \in \mathbb{N}}$, where $\mu^{r}$ is a proper solution to (10). $\mathrm{P}^{0}$-a.s.,

Uniqueness. Fix $r$ and $\tilde{r}$ to be as above. There exists a solution $\mu^{\tilde{r}}$ to (10). Then we have,

$$
\left\langle\mu^{\tilde{r}}, I\right\rangle \circ \theta=\left\langle\Phi^{\tilde{r}}\left(\mu^{\tilde{r}}+\delta_{\sigma}, \xi\right), I\right\rangle=\left[\left\langle\mu^{\tilde{r}}, I\right\rangle+\sigma-K_{r} \xi\right]^{+}
$$

hence, $\left\langle\mu^{\tilde{r}}, I\right\rangle$ equals $W^{K_{r}}, \mathrm{P}^{0}$-a.s. Moreover, on $\left\{\left\langle\mu^{r}, I\right\rangle \leq W^{K^{r}}\right\}$, we have, in view of Lemma 2 ,

$$
\left\langle\mu^{r}, I\right\rangle \circ \theta \leq\left\langle\Phi^{\tilde{r}}\left(\mu^{r}+\delta_{\sigma}, \xi\right), I\right\rangle=\left[\left\langle\mu^{r}, I\right\rangle+\sigma-K_{r} \xi\right]^{+} \leq W^{K_{r}} \circ \theta \quad \mathrm{P}^{0} \text {-a.s.; }
$$

thus, the event $\left\{\left\langle\mu^{r}, I\right\rangle \leq W^{K_{r}}\right\}$ is $\theta$-contracting. Moreover,

$$
\mathrm{P}^{0}\left[\left\langle\mu^{r}, I\right\rangle \leq W^{K_{r}}\right] \geq \mathrm{P}^{0}\left[\left\langle\mu^{r}, I\right\rangle=0\right]>0,
$$

as another consequence of (11) and the ergodic lemma. Therefore, $\left\langle\mu^{r}, I\right\rangle \leq W^{K_{r}}, \mathrm{P}^{0}$-a.s., so that

$$
\mathfrak{A}_{n} \subseteq\left\{\left\langle\mu^{r}, I\right\rangle \circ \theta^{n}=0\right\}=\left\{\mu^{r} \circ \theta^{n}=\tilde{0}\right\} .
$$

Consequently, $\left\{\mathfrak{A}_{n}\right\}_{n \in \mathbb{N}}$ is a stationary sequence of renovating events of length 1 for $\left\{\mu^{r} \circ \theta^{n}\right\}_{n \in \mathbb{N}}$ for any solution $\mu^{r}$ of $(10)$ associated to the rate $r$. Since $\mathrm{P}^{0}\left[\mathfrak{A}_{0}\right]>0$, there exists a unique solution to (10) in view of Remark 2.5.3 of [1].

In particular, we have the following corollary.

Corollary 2. Under condition (11), for any $\zeta$ such that $\langle\zeta, I\rangle \leq W^{K_{r}}, \mathrm{P}^{0}$-a.s.,

(i) $\left\{X_{n}^{[N(\zeta)]}\right\}_{n \in \mathbb{N}}$ converges with strong backwards coupling to $N\left(\mu^{r}\right)$,

(ii) $\left\{W_{n}^{[\langle\zeta, I\rangle]}\right\}_{n \in \mathbb{N}}$ converges with strong backwards coupling to $\left\langle\mu^{r}, I\right\rangle$.

\section{Appendix A. Proofs of monotonicity}

For easy checking, in this appendix we present the details of the proofs of Lemmas 3 and 4.

Proof of Lemma 3. We again fix $x \in \mathbb{R}+$ and $\mu, v \in \mathcal{M}$ such that $\mu \preceq v$. Whenever $i^{r}(\mu, x)<N(\mu)$ (otherwise $\Phi^{r}(\mu, x)=\tilde{0}$ ), we have

$$
\begin{array}{r}
\quad \sum_{j=1}^{N(v)-N(\mu)+i^{r}(\mu, x)} \alpha_{j}(v)\left(\frac{1}{r(N(v)-j+1)}-\frac{1}{r(N(v)-j)}\right) \\
\geq \sum_{j=1}^{i^{r}(\mu, x)} \alpha_{j}(\mu)\left(\frac{1}{r(N(\mu)-j+1)}-\frac{1}{r(N(\mu)-j)}\right),
\end{array}
$$


which implies that

$$
\begin{aligned}
\alpha_{N(v)-N(\mu)+i^{r}(\mu, x)+1}(v) \geq & \alpha_{i^{r}(\mu, x)+1}(\mu) \\
\geq & r\left(N(\mu)-i^{r}(\mu, x)\right) \\
& \quad \times\left(x-\sum_{j=1}^{i^{r}(\mu, x)} \alpha_{j}(\mu)\left(\frac{1}{r(N(\mu)-j+1)}-\frac{1}{r(N(\mu)-j)}\right)\right) \\
\geq & \gamma_{N(v)-N(\mu)+i^{r}(\mu, x)+1}^{r}(v, x) .
\end{aligned}
$$

This means that $i_{0}(v, x) \leq N(v)-N(\mu)+i_{0}(\mu, x)$, i.e. $N\left(\Phi^{r}(\mu, x)\right) \leq N\left(\Phi^{r}(v, x)\right)$. Hence, in view of (4) we have

$$
\begin{aligned}
\gamma(\mu, \xi) & =\gamma_{i^{r}(\mu, \xi)+1}^{r}(\mu, x) \\
& \geq \gamma_{\left(i^{r}(v, \xi)+N(\mu)-N(v)\right)^{+}+1}^{r}(\mu, x) \\
& \geq r\left(N(v)-i^{r}(v, x)\right)\left(x-\sum_{j=1}^{i^{r}(v, x)} \alpha_{j}(v)\left(\frac{1}{r(N(v)-j+1)}-\frac{1}{r(N(v)-j)}\right)\right) \\
& =\gamma^{r}(v, x),
\end{aligned}
$$

which clearly implies that $\Phi^{r}(\mu, x) \preceq \Phi^{r}(\nu, x)$.

Proof of Lemma 4. We now fix $\mu \in \mathcal{M}$ and $x \in \mathbb{R}_{+}$. For any two rate functions $r$ and $\tilde{r}$ such that $r(i) \leq \tilde{r}(i)$ for any $i \in \mathbb{N}^{*}$, the induction formula, (2), straightforwardly shows that $i^{r}(\mu, x) \geq i^{\tilde{r}}(\mu, x)$, i.e. $N\left(\Phi^{r}(\mu, x)\right) \leq N\left(\Phi^{\tilde{r}}(\mu, x)\right)$. Hence, as in the previous proof, $\gamma^{r}(\mu, x) \leq \gamma^{\tilde{r}}(\mu, x)$.

\section{Acknowledgement}

The author would like to warmly thank Bryan Renne for useful discussions in Dublin.

\section{References}

[1] Baccelli, F. ANd Brémaud, P. (2002). Elements of Queueing Theory. 2nd edn. Springer, Berlin.

[2] Billingsley, P. (1968). Convergence of Probability Measures. John Wiley, New York.

[3] Borovkov, A. A. (1976). Stochastic Processes in Queueing Theory. Springer, New York.

[4] Borovkov, A. A. And Foss, S. (1992). Stochastically recursive sequences and their generalizations. Siberian Adv. Math., 2, 16-81.

[5] Decreusefond, L. And Moyal, P. (2007). A functional central limit theorem for the M/GI/ $\infty$ queue. To appear in Ann. Appl. Prob.

[6] Loynes, R. M. (1962). The stability of queues with non-independent interarrivals and service times. Proc. Camb. Phil. Soc. 58, 497-520.

[7] Moyal, P. (2007). Construction of a stationary FIFO queue with impatient customers. Preprint. Available at http://arxiv.org/abs/0802.2495. 